\section{Tumor inflamatorio miofibroblástico peritoneal. Respuesta favorable asociada a infliximab. Caso clínico}

\author{
DANIELA GRÜNHOLZ ${ }^{1}$, FRANCO APPIANI ${ }^{2,3, a}$, CONSTANZAABARCA ${ }^{2,3, a}$, \\ MARÍA MANRÍQUEZ ${ }^{4}$, JORGE PINILLA ${ }^{4}$, EDUARDO WAINSTEIN ${ }^{5}$
}

\section{Peritoneal myofibroblastic tumor successfully treated with infliximab. Report of one case}

Myofibroblastic tumor (MIT) is characterized by the infiltration of different organs, most commonly the lungs, with nodular lesions composed of myofibroblasts and inflammatory cells, which can be identified by specific patterns in the immunohistochemical studies. When it involves the peritoneum it is difficult to eradicate, tends to relapse and it has an invasive behavior, requiring its differentiation from peritoneal carcinomatosis. Treatment may be surgical excision, the use of non-steroidal anti-inflammatory drugs (NSAIDs) and glucocorticoids. We report a 30 years old female with an unremarkable medical history, presenting with abdominal pain and progressive abdominal distention. A CT scan revealed multiple peritoneal nodular lesions. A surgical biopsy was reported as a myofibroblast and inflammatory cell infiltrate. Immunohistochemical staining was consistent with MIT. Given the extensive involvement of the peritoneum surgical therapy was not considered appropriate and treatment with NSAID and glucocorticoids was started. No response was observed after 6 months, therefore infliximab therapy was started. After 10 months of follow-up the patient is well, returned to normal life, ascites improved and resolved and CT scan showed partial regression or stabilization of the lesions.

(Rev Med Chile 2015; 143: 943-947)

Key words: Anti-inflammatory agents; Inflammatory neoplasms; Infliximab; Non-steroidal clooxygenase-2 inhibitors.
${ }^{1}$ Servicio de Medicina Interna y Reumatología, Hospital Militar de Santiago. Facultad de Medicina Universidad de Valparaíso. ${ }^{2}$ Facultad de Medicina Universidad de Valparaíso. ${ }^{3}$ Hospital Militar de Santiago. ${ }^{4}$ Servicio de Anatomía Patológica, Hospital Militar de Santiago ${ }^{5}$ Servicio Reumatología, Hospital Militar de Santiago.

aResidente Medicina Interna.

Recibido el 3 de octubre de 2014, aceptado el 30 de abril de 2015.

Correspondencia a:

Dra. Daniela Grünholz Gambi danielagru@gmail.com
E 1 tumor inflamatorio miofibroblástico (TIM) o pseudotumor inflamatorio es una enfermedad poco frecuente, de etiología desconocida, caracterizada por lesiones tumorales en diversos órganos y tejidos blandos ${ }^{1}$. La ubicación más frecuentemente descrita es la pulmonar, siendo la abdominal poco común. La mayoría de las ocasiones es de comportamiento benigno, ubicación única y de simple resolución quirúrgica. Sin embargo, en ocasiones es difícil de diferenciar de una neoplasia maligna debido a su capacidad de invasión local y tendencia a recurrir ${ }^{2-4}$. En escasas oportunidades su presentación es multifocal y metastásica de difícil alcance terapéutico.
La histología se caracteriza por la presencia de miofibroblastos y células inflamatorias ${ }^{1,5,6}$. Se ha discutido si su origen es neoplásico o naturaleza post inflamatoria. El diagnóstico diferencial incluye una gran variedad de lesiones neoplásicas y el tratamiento quirúrgico es habitualmente curativo, aunque se han descrito casos con resolución espontánea ${ }^{7,8,9}$.

A continuación se presenta un caso de TIM de ubicación peritoneal y comportamiento agresivo, cuya resolución quirúrgica no fue factible, comportándose también refractario a terapia médica convencional. Se utilizó infliximab con una respuesta favorable tanto clínica como imagenológica. 


\section{Caso clínico}

Mujer de 30 años previamente sana que consulta en octubre de 2012 por cuadro de dolor y distensión abdominal progresiva. Ecografía abdominal de urgencia mostró hígado graso y abundante ascitis. Hematocrito 42\%, hemoglobina de $13 \mathrm{~g} / \mathrm{dl}, 13.600$ leucocitos y 582.000 plaquetas. Proteína C reactiva $112 \mathrm{mg} / \mathrm{L}$ (VR: hasta $5 \mathrm{mg} / \mathrm{L}$ ), perfil bioquímico, perfil hepático, función renal y electrolitos plasmáticos normales.

Se realiza tomografía axial computada de abdomen que mostró discreto derrame pleural izquierdo, con aumento de densidad difusa del tejido adiposo mesoperitoneal asociado a la presencia de implantes peritoneales de tipo metastásico y abundante ascitis.

El estudio del líquido ascítico evidenció: Adenosindeaminasa: 7,35 (VR: hasta $32 \mathrm{UI} / \mathrm{L}$ ), glucosa $115 \mathrm{mg} / \mathrm{dl}$, proteínas $6 \mathrm{~g} / \mathrm{dl}$, recuento de leucocitos $1.100 \mathrm{cel} / \mathrm{mm}^{3}$ con $91 \%$ de mononucleares y $9 \%$ de polimorfonucleares. Resultados negativos para la baciloscopia, cultivo de Koch y cultivo corriente.

Dados estos hallazgos se solicita además antígeno carcinoembrionario el cual resultó menor a $0,5 \mathrm{ng} / \mathrm{ml}$, alfafetoproteínas menor a $1,3 \mathrm{ng} / \mathrm{mL}$ y gonadotrofina coriónica menor de $2 \mathrm{mUI} / \mathrm{Ml}$.

Debido a la ausencia de diagnóstico etiológico se decide realizar laparotomía exploradora, la cual evidenció múltiples nódulos en peritoneo, abundante líquido ascítico de aspecto turbio, destacando ovarios y útero de aspecto macroscópico normal.
Se realiza análisis anatomopatológico de tres lesiones peritoneales, evidenciándose a la microscopía tejido adiposo extensamente comprometido con infiltrado inflamatorio crónico linfocitario y plasmocelular, polimorfonucleares neutrófilos y fibrosis hialina. Destaca una lesión compuesta de miofibroblastos de forma elongada con cromatina pálida y pequeño nucléolo puntiforme (Figura 1).

La inmunohistoquímica resultó negativa para ALK, calretinina, CD30, CK5-6 y desmina, y fue positiva. Resultó positiva para actina de músculo liso y Ki67 (en 5-10\%), compatible con diagnóstico de TIM.

Dado que el manejo quirúrgico en este contexto podría asociarse a gran morbi-mortalidad, por extensión de las lesiones peritoneales se plantea terapia médica con prednisona $40 \mathrm{mg}$ diarios asociada a celecoxib $400 \mathrm{mg}$ al día, que recibió por 6 meses. La paciente evoluciona desfavorablemente, pues el dolor y distensión abdominal aumentan lo que la inhabilita para ejercer sus funciones laborales (Imagen 2). Se realiza tomografía de control donde se observa aumento del tamaño y número de lesiones peritoneales asociado a mayor ascitis, derrame pleural leve y nuevas lesiones en hígado y pleura.

Frente al comportamiento agresivo se realiza nuevamente estudio histológico y se realiza nueva laparotomía exploradora. Los análisis anatomopatológico e inmunohistoquímico evidenciaron los mismos resultados.

Dada ausencia de alto índice mitótico en el análisis histológico se desestima manejo con quimioterapia.
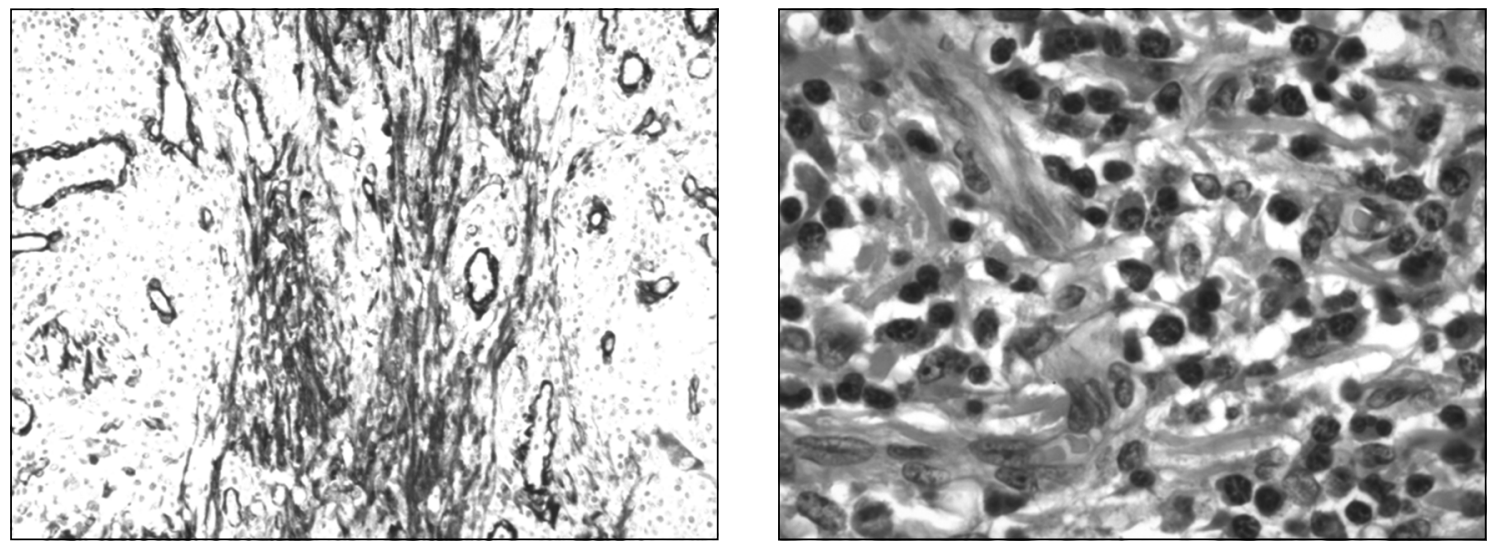

Figura 1. Imagen izquierda, ACTINA 10X. Se observan abundantes células miofibroblasticas positivas para actina. Imagen derecha H\&E 40X. Se observan miofibroblastos con abundantes células plasmáticas. 

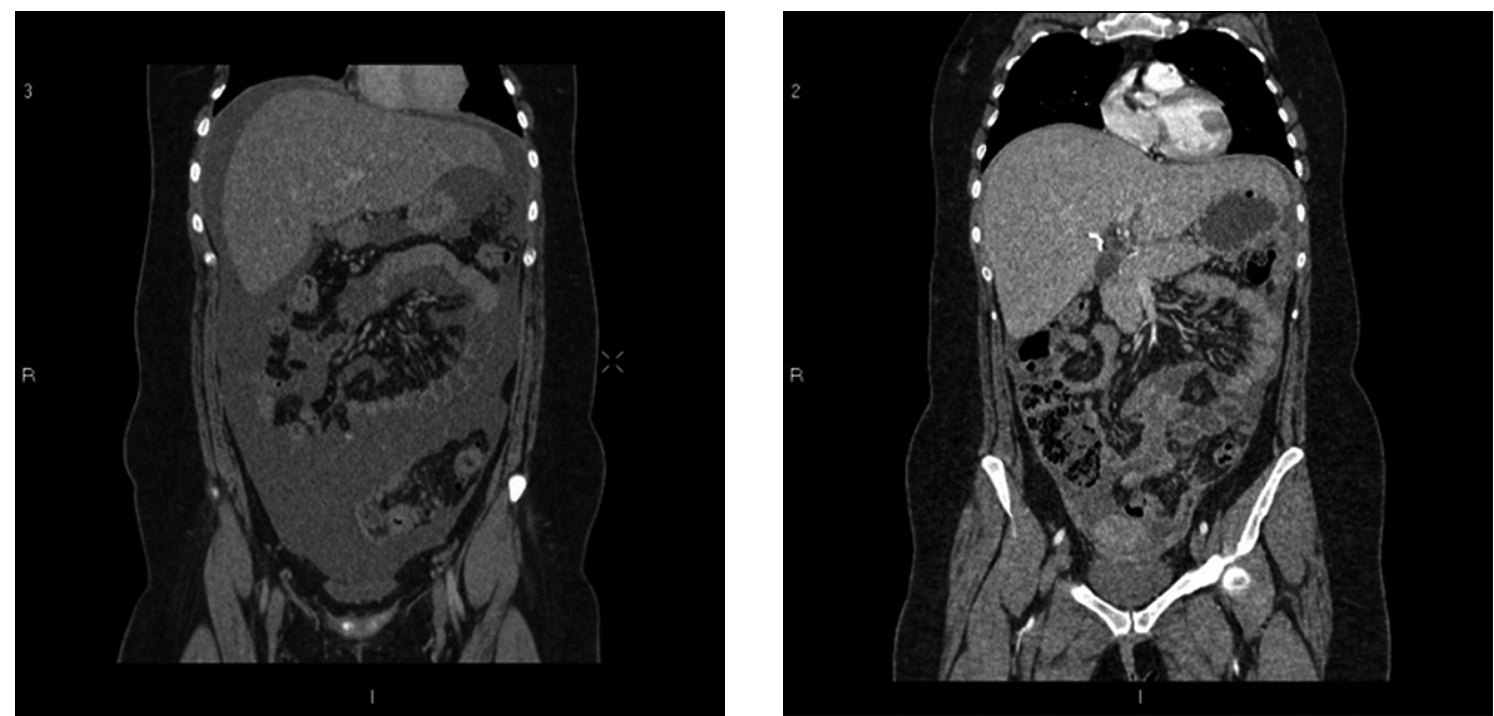

Figura 2. Tomografía axial computada de abdomen y pelvis en corte coronal. Imagen izquierda, hallazgos previos a tratamiento, se observa abundante ascitis y engrosamiento peritoneal difuso. Imagen derecha, posterior a 9 meses post inicio de infliximab. Se observa reducción de la ascitis, sin nuevos implantes peritoneales.

Tras revisión de literatura se plantea uso infliximab a partir de un caso similar con buenos resultados. Posterior a la discusión del caso con el comité de ética de nuestro centro, además de obtener consentimiento de la paciente, se inicia infliximab intravenoso en dosis de $300 \mathrm{mg}$ en semana $0,2,6$ y luego cada 8 semanas. Previo al uso de infliximab se realizó estudio para virus de hepatitis $\mathrm{B}$, hepatitis $\mathrm{C}$ e inmunodeficiencia humana y estudio para TBC latente con QuantiFERON ${ }^{\circledR}$, todos con resultados negativos.

El seguimiento de la paciente a los 10 meses demuestra disminución del dolor abdominal y distensión, logrando reintegrarse a su actividad laboral. En la tomografía de control a los 9 meses se observa estabilidad de las lesiones tumorales, ausencia de nuevas lesiones y regresión de la ascitis (Figura 2).

\section{Discusión}

El tumor inflamatorio miofibroblástico (TIM) o pseudotumor inflamatorio es una enfermedad caracterizada por la aparición de lesiones compuestas por miofibroblastos e infiltración linfoplasmocitaria. Se le ha denominado de diferentes formas: pseudotumor inflamatorio, granuloma de células plasmáticas, fibrosarcoma inflamatorio, fibrohistiocitoma, pólipo fibroide inflamatorio entre otras ${ }^{2}$. Descrito originalmente en 1937 en un caso con afección pulmonar, desde esa fecha se ha reportado en diferentes zonas anatómicas ${ }^{3}$.

Este tumor se observa con mayor frecuencia en niños y en las primeras 2 a 3 décadas de la vida. Las localizaciones más frecuentes son pulmón, mesenterio y epiplón ${ }^{2}$.

Microscópicamente el tumor está formado por células fusiformes que corresponden a miofibroflastos mezclados con un componente inflamatorio crónico a base de células plasmáticas, linfocitos $\mathrm{y}$, en ocasiones, histiocitos ${ }^{4}$.

En la inmunohistoquímica las células suelen expresar difusamente vimentina, actina y desmina (aunque hay casos con resultado negativo) y son negativos para cKIT. Hasta el $71 \%$ son positivos para ALK-1 marcador altamente específico de TIM pero no sensible $2,5,6$.

El diagnóstico diferencial debe hacerse con lesiones malignas tales como sarcoma miofibroblástico, carcinoma de células fusadas, y lesiones benignas como leiomiomas, tumor fibroso solitario y fascitis nodular. Estos diagnósticos se diferencian por su análisis patológico e inmunohistoquímico ${ }^{19}$.

La etiopatogenia aún no está bien establecida pero se plantean dos hipótesis fundamentales, 
una inflamatoria y otra neoplásica. La primera se respalda en sus características inflamatorias a la histología planteándose como una respuesta exagerada a una infección, trauma o cirugía ${ }^{4,11}$. Además, se ha descrito sobreexpresión de mediadores de la angiogénesis tales como el factor de crecimiento endotelial vascular (VEGF) y la ciclooxigenasa 2 (COX-2), apoyando también su estirpe inflamatoria. Por otro lado, el componente neoplásico se basa en ciertos casos de comportamiento agresivo donde se presenta de manera diseminada, metastásica y con recurrencia local posterior al tratamiento ${ }^{2,3}$, aunque se han descrito casos con resolución espontánea ${ }^{7}$.

En al menos 50\% de los casos puede pesquisarse reordenamientos en el gen ALK, (quinasa de linfoma anaplástico) ubicado en el cromosoma $2 \mathrm{p} 23$, hecho que también estaría a favor de su conformación neoplásica ${ }^{12}$.

Se han tratado de establecer características que puedan predecir un peor pronóstico, recurrencia y probabilidad de metástasis, las cuales son sexo femenino, edad mayor a 25 años, ubicación abdomino-pélvica, gran tamaño, masa multinodular, resección incompleta y ALK negativo ${ }^{2}$, todas características presentes en el caso expuesto.

Con relación al tratamiento, la opción quirúrgica con intención curativa es la terapia de elección en casos sin regresión tumoral espontánea. Sin embargo, en casos donde la cirugía es impracticable ya sea por los riesgos o porque no habrá resección total, se han investigado diversas opciones. Estas se basan en la etiopatogenia de este tumor y poseen evidencia no mayor a sólo reporte de casos.

La radioterapia y la quimioterapia tienen roles poco claros como modalidades complementarias $^{2,10}$. Algunos reportes han publicado buena respuesta en pacientes con uso de antineoplásicos como vinblastina, cisplatino y metotrexato en lesiones irresecables ${ }^{3}$. En nuestro caso, por ausencia de claros componentes de neoplasia y bajo índice mitótico al análisis histológico, se desestimó como alternativa terapéutica el uso de quimioterapia.

Basados en la hipótesis que apoya el componente inflamatorio como patogenia esencial, se han publicado varios casos con uso de anti-inflamatorios no esteroidales y prednisona para el tratamiento alternativo y coadyuvancia al tratamiento quirúrgico ${ }^{8,12-16}$.

El modo de acción de los fármacos anti-inflamatorios sigue siendo desconocido. Se postula un efecto inhibitorio sobre la angiogénesis y la proliferación celular a través de la inducción de apoptosis de fibroblastos $y$, por tanto, pueden inhibir la vascularización tumoral, la proliferación endotelial, y el crecimiento del tumor ${ }^{5}$. Basados en las propiedades de los inhibidores de la COX-2 y en los casos reportados en la literatura ${ }^{12-16}$ se decidió indicarlos en este caso pero no hubo respuesta clínica ni radiológica.

Germadinis y colaboradores reportan en el año 2005 un caso similar tratado con infliximab en dosis de $300 \mathrm{mg}$ en la semama 0, 2, 6 y luego cada 8 semanas con regresión clínica tumoral ${ }^{17}$. Según nuestro conocimiento, no hay hasta la fecha otros reportes de manejo de TIM con este tipo de fármaco.

Dentro de los muchos efectos del infliximab se encuentra producción de apoptosis celular, inhibición del factor de necrosis tumoral (TNF) y disminución de la inflamación, por diferentes vías. Se usa ampliamente en enfermedades reumatológicas y enfermedad de Crohn y su seguridad está ampliamente demostrada.

Se decidió en nuestro caso utilizar el mismo esquema de tratamiento publicado. La paciente presentó una evolución favorable tanto clínica como radiológica, con regresión de la ascitis, mejoría de la calidad de vida y retorno a sus actividades laborales.

Se lleva un seguimiento hasta la fecha de 10 meses, con la paciente clínicamente en buenas condiciones y regresión completa de la ascitis en las imágenes. No ha habido reacciones adversas a la terapia.

\section{Conclusión}

En nuestro conocimiento, este es el segundo caso publicado de respuesta clínica favorable con anti-TNF en un paciente portador de TIM peritoneal. Pensamos que el infliximab puede ser considerado una opción terapéutica en pacientes con TIM irresecables.

\section{Referencias}

1. Kosma L, Khaldi L, Galani P, Mytas D, Lafoyianni S. A Rare Case of aninflammatory myofibroblastic tumor in a middle-aged female. Case Reports in Oncological Medicine 2012; 10: 4 . 
2. Fragoso A, Eloy C, Estevão-Costa J, Campos M, Farinha $\mathrm{N}$, Lopes J. Abdominal inflammatory myofibroblastic tumor: a clinicopathologic study with reappraisal of biologic behavior. Journal of Pediatric Surgery 2011; 46: 2076-82.

3. Tao YL, Wang ZJ, Han JG, Wei P. Inflammatory myofibroblastic tumor successfully treated with chemotherapy and nonsteroidals: A case report. World J Gastroenterol 2012; 18 (47): 7100-3.

4. Kye BH, Kim HJ, Kang SG, Yoo C, Cho HM. A case of inflammatory myofibroblastic tumor originated from the greater omentum in Young adult. J Korean Surg Soc 2012; 82: 380-4.

5. Coffin CM, Hornick JL, Fletcher CD. Inflammatory myofibroblastic tumor: comparison of clinicopathologic, histologic, and immunohistochemical featuresincluding ALK expression in atypical and aggressive cases. Am J Surg Pathol 2007; 31 (4): 509-20.

6. Coffin CM, Watterson J, Priest JR, Dehner LP. Extrapulmonary inflammatory myofibroblastic tumor (inflammatory pseudotumor). A clinicopathologic and immunohistochemical study of 84 cases. Am J Surg Pathol 1995; 19 (8): 859-72.

7. Zhao JJ. Intra-abdominal inflammatory myofibroblastic tumor: spontaneous regression. World J Gastroenterol 2014; 20 (37): 13625-31.

8. Calaway AC. Spontaneous resolution of inflammatory myofibroblastic tumor of the kidney. Can J Urol 2014; 21 (6): 7582-5.

9. Qiu X, Montgomery E, Sun B. Inflammatory Myofibroblastic tumor and low-grade myofibroblastic sarcoma: a comparative study of clinicopathologic features and further observations on the immunohistochemical profile of myofibroblasts. Hum Pathol 2008; 39 (6): 846-56.

10. Diop B, Konate I, Ka S, Sall I, Fall D, Dieng M, et al. Mesenteric myofibroblastic tumor: NSAID therapy after incomplete resection. J Visc Surg 2011; 148 (4): 311-4.

11. Singhal M, Ramanathan S, Das A, Singh G, Bagga R, Khandelwal N. Omental inflammatory myofibroblastic tumour mimicking peritoneal carcinomatosis. Cancer Imaging 2011; 11: 19-22.

12. Chávez C, Hoffman M. Celecoxib treatment of inflammatory myofibroblastic tumor. Oncology Letters 2013; 5: 1672-6.

13. Applebaum H, Kieranb M, Cripec T, Coffind C, Collinse M, Kaipainenf A, et al. The rationale for nonsteroidal anti-antinflammatory drug therapy for inflammatory myofibroblastic tumors: a Children's Oncology Groupstudy. Journal of Pediatric Surgery 2005; 40: 999-1003.

14. Mattei P, Barnaby K. Rapid regression of duodenal inflammatory myofibroblastic tumor after intravenous ketorolac: case report and review of the literature. Journal of Pediatric Surgery 2008; 43: 1196-9.

15. Bertocchini A, Lo Zupone C, Callea F, Gennari F, Serra A, Monti L. Unresectable multifocal omental and peritoneal inflammatory myofibroblastic tumor in a child: revisiting the role of adjuvanttherapy. J Pediatr Surg 2011; 46 (4): 17-21.

16. Kusunoki-Nakamoto F, Matsukawa T, Tanaka M, Miyagawa T, Yamamoto T, Shimizu J, et al. Successful treatment of an unresectable inflammatory myofibroblastic tumor of the frontal bone using a cyclooxygenase-2 inhibitor and methotrexate. Intern Med 2013; 52 (5): 623-8.

17. Germanidis G, Xanthakis I, Tsitouridis I, Zaramboukas T, Kiskinis D, Konstantaras C, et al. Regression of inflammatory myofibroblastic tumor of the gastrointestinal tract under infliximab treatment. Digestive Deaseases and Science 2005; 50 (2): 262-5.

18. Patnana M, Sevrukov AB, Elsayes KM, Viswanathan C, Lubner M, Menias CO. Inflammatory pseudotumor: thegreatmimicker. AJR Am J Roentgenol 2012; 198 (3): 217-27.

19. Volker HU, Scheich M, Holler S, Strobel P, Hagen R, Hermenlink HK, et al. Differential diagnosis of laryngeal spindle cell carcinoma and inflammatory tumour: report of two cases with similar morphology. Diagn Pathol 2007; 2: 1-7. 